\title{
Bayesian Approximation Techniques of Inverse Exponential Distribution with Applications in Engineering
}

\author{
Kawsar Fatima, S.P Ahmad \\ Department of Statistics, University of Kashmir, Srinagar, India
}

Received: 06 February 2018; Accepted: 15 March 2018; Published: 08 April 2018

\begin{abstract}
The present study is concerned with the estimation of Inverse Exponential distribution using various Bayesian approximation techniques like normal approximation, Tierney and Kadane (T-K) Approximation. Different informative and non-informative priors are used to obtain the Baye's estimate of Inverse Exponential distribution under different approximation techniques. A simulation study has also been conducted for comparison of Baye's estimates obtained under different approximation using different priors.
\end{abstract}

Index Terms: Bayesian Estimation, Prior Distribution, Normal Approximation, T-K Approximation.

(C) 2018 Published by MECS Publisher. Selection and/or peer review under responsibility of the Research Association of Modern Education and Computer Science

\section{Introduction}

The Inverse Exponential distribution was introduced by Keller and Kamath (1982). Due to its inverted bathtub failure rate, it is significant competitive model for the Exponential distribution. A comprehensive description of this model is given by Lin et al (1989) as a lifetime model. Consider a random variable $X$ following an exponential distribution and then the variable $Y=\frac{1}{X}$ will have an inverted exponential distribution (IED). Thus, if $\mathrm{X}$ follows the Inverse Exponential distribution with a scale parameter $\lambda(\lambda>0)$, then the cumulative density function (cdf) and the probability density function (pdf) of the model are respectively given by:

* Corresponding author.

E-mail address: kawsarfatima@gmail.com 


$$
f(x)=\frac{\lambda}{x^{2}} e^{\frac{-\lambda}{x}} ; x>0, \lambda>0
$$

The cdf of $\mathrm{X}$ is:

$$
F(x)=e^{\frac{-\lambda}{x}} ; x>0, \lambda>0
$$

Inverted exponential distribution as a life distribution model from a Bayesian viewpoint was considered by Sanku Dey (2007) while Gyan Prakash (2012) obtained the estimation of the Inverted exponential distribution by using symmetric and asymmetric loss functions. Singh et al (2015) discussed the estimation of stress strength reliability parameter of inverted exponential distribution. They obtained Bayes estimator for parameters of inverted exponential distribution by using informative and non-informative priors. They also compared the classical method with Bayesian method through the simulation study.

\section{Material and Methods}

The Bayesian paradigm is conceptually simple and probabilistically elegant. Sometimes posterior distribution is expressible in terms of complicated analytical function and requires intensive calculation because of its numerical implementations. It is therefore useful to study approximate and large sample behavior of posterior distribution. Thus, our present study focuses to obtain the estimates of the parameter of inverted exponential distribution using two Bayesian approximation techniques i.e. normal approximation and T-K approximation.

\section{Normal Approximation}

The basic result of the large sample Bayesian inference is that the posterior distribution of the parameter approaches a normal distribution. If the posterior distribution $p(\lambda \mid x)$ is unimodal and roughly symmetric, it is convenient to approximate it by a normal distribution centered at the mode; that is logarithm of the posterior is approximated by a quadratic function, yielding the approximation

$$
p(\lambda \mid x) \sim N\left(\hat{\lambda},[I(\hat{\lambda})]^{-1}\right)
$$

where

$$
I(\hat{\lambda})=-\frac{\partial^{2} \log P(\lambda \mid x)}{\partial \lambda^{2}}
$$

If the mode, $\hat{\lambda}$ is in the interior parameter space, then $I(\lambda)$ is positive; if $\hat{\lambda}$ is a vector parameter, then $I(\lambda)$ is a matrix.

Some good sources on the topic is provided by Ahmad et.al $(2007,2011)$ discussed Bayesian analysis of exponential distribution and gamma distribution using normal and Laplace approximations. Sultan et al. (2015a, $2015 \mathrm{~b}$ ) obtained the Baye's estimates under different informative and non-informative priors of shape parameter of Topp-Leone and Kumaraswamy Distribution using Bayesian approximation techniques. 
In our study the normal approximations of Inverse Exponential distribution under different priors is to be obtained as under:

The likelihood function of (1) for a sample of size $\mathrm{n}$ is given as

$$
L(\underline{x} \mid \lambda)=\lambda^{n} \sum_{i=1}^{n} x_{i}^{-2} e^{-\lambda \sum_{i=i}^{n} x_{i}^{-1}} .
$$

Under Quasi prior $g(\lambda)=\frac{1}{\lambda^{d}}, d \geq 0$, the posterior distribution for $\lambda$ is as

$$
P(\lambda \mid x) \propto \lambda^{n-d} e^{-\lambda T}, \text { where } T=\sum_{i=i}^{n} x_{i}^{-1}
$$

The first derivative is

$$
\frac{\partial}{\partial \lambda} \log P(\lambda \mid x)=\frac{n-d}{\lambda}-T
$$

from which the posterior mode is obtained as $\hat{\lambda}=\frac{(n-d)}{T}$.

To construct the approximation, we need the second derivatives of the log-posterior density,

$$
\log P(\lambda \mid x)=\log \text { constant }+(n-d) \log \lambda-\lambda T .
$$

The second derivative of the log-posterior density is

$$
\frac{\partial^{2} \log P(\lambda \mid x)}{\partial \lambda^{2}}=\frac{-(n-d)}{\lambda^{2}}
$$

and hence, negative of Hessian is

$$
I(\hat{\lambda})=-\frac{\partial^{2} \log P(\lambda \mid x)}{\partial \lambda^{2}}=\frac{T^{2}}{(n-d)} \Rightarrow[I(\hat{\lambda})]^{-1}=\frac{(n-d)}{T^{2}} .
$$

Thus, the posterior distribution can be approximated as

$$
p(\lambda \mid x) \sim N\left(\frac{(n-d)}{T}, \frac{(n-d)}{T^{2}}\right) .
$$

Under extension of Jeffrey's prior $g(\lambda)=\frac{1}{\lambda^{2 c_{1}}}, c_{1} \in R^{+}$, the posterior distribution for $\lambda$ is as 
$P(\lambda \mid x) \propto \lambda^{n-2 c_{1}} e^{-\lambda T}$, where $T=\sum_{i=i}^{n} x_{i}^{-1}$

$\log P(\lambda \mid x)=\log$ constant $+\left(n-2 c_{1}\right) \log \lambda-\lambda T$.

The first derivative is

$$
\frac{\partial}{\partial \lambda} \log P(\lambda \mid x)=\frac{n-2 c_{1}}{\lambda}-T
$$

from which the posterior mode is obtained as $\hat{\lambda}=\frac{\left(n-2 c_{1}\right)}{T}$.

The second derivative of the log-posterior density is

$$
\frac{\partial^{2} \log P(\lambda \mid x)}{\partial \lambda^{2}}=\frac{-\left(n-2 c_{1}\right)}{\lambda^{2}}
$$

and hence, negative of Hessian is

$$
I(\hat{\lambda})=-\frac{\partial^{2} \log P(\lambda \mid x)}{\partial \lambda^{2}}=\frac{T^{2}}{\left(n-2 c_{1}\right)} \Rightarrow[I(\hat{\lambda})]^{-1}=\frac{\left(n-2 c_{1}\right)}{T^{2}}
$$

Thus, the posterior distribution can be approximated as

$$
p(\lambda \mid x) \sim N\left(\frac{\left(n-2 c_{1}\right)}{T}, \frac{\left(n-2 c_{1}\right)}{T^{2}}\right)
$$

Under the Pareto1 prior $g(\lambda)=b c^{b} \lambda^{-(b+1)}, c, b>0 ; \lambda>0$ where $\mathrm{b}$ and $\mathrm{c}$ are the known hyper parameters, the posterior distribution for $\lambda$ is as

$$
P(\lambda \mid x) \propto \lambda^{n-b-1} e^{-\lambda T}, \text { where } T=\sum_{i=i}^{n} x_{i}^{-1}
$$

$\log P(\lambda \mid x)=\log$ constant $+(n-b-1) \log \lambda-\lambda T$.

The first derivative is

$$
\frac{\partial}{\partial \lambda} \log P(\lambda \mid x)=\frac{(n-b-1)}{\lambda}-T
$$

from which the posterior mode is obtained as $\hat{\lambda}=\frac{(n-b-1)}{T}$. 
The second derivative of the log-posterior density is

$$
\frac{\partial^{2} \log P(\lambda \mid x)}{\partial \lambda^{2}}=\frac{-(n-b-1)}{\lambda^{2}}
$$

and hence, negative of Hessian is

$$
I(\hat{\lambda})=-\frac{\partial^{2} \log P(\lambda \mid x)}{\partial \lambda^{2}}=\frac{T^{2}}{(n-b-1)} \Rightarrow[I(\hat{\lambda})]^{-1}=\frac{(n-b-1)}{T^{2}} .
$$

Thus, the posterior distribution can be approximated as

$$
p(\lambda \mid x) \sim N\left(\frac{(n-b-1)}{T}, \frac{(n-b-1)}{T^{2}}\right)
$$

Under the Inverse Levy prior $g(\lambda)=\sqrt{\frac{a}{2 \pi}} \lambda^{-\frac{1}{2}} e^{-\frac{a \lambda}{2}} ; a>0 ; \lambda>0$, where $a$ is the known hyper parameter, thus the posterior distribution for $\lambda$ is as

$$
P(\lambda \mid x) \propto \lambda^{n-1 / 2} e^{-\lambda(T+a / 2)}, \text { where } T=\sum_{i=i}^{n} x_{i}{ }^{-1}
$$

$\log P(\lambda \mid x)=\log$ constant $+(n-1 / 2) \log \lambda-\lambda(T+a / 2)$

The first derivative is

$$
\frac{\partial}{\partial \lambda} \log P(\lambda \mid x)=\frac{(n-1 / 2)}{\lambda}-\left(\frac{a}{2}+T\right)
$$

from which the posterior mode is obtained as $\hat{\lambda}=\frac{(n-1 / 2)}{\left(\frac{a}{2}+T\right)^{2}}$.

The second derivative of the log-posterior density is

$$
\frac{\partial^{2} \log P(\lambda \mid x)}{\partial \lambda^{2}}=\frac{-(n-1 / 2)}{\lambda^{2}}
$$

and hence, negative of Hessian is 


$$
I(\hat{\lambda})=-\frac{\partial^{2} \log P(\lambda \mid x)}{\partial \lambda^{2}}=\frac{\left(\frac{a}{2}+T\right)^{2}}{(n-1 / 2)} \Rightarrow[I(\hat{\lambda})]^{-1}=\frac{(n-1 / 2)}{\left(\frac{a}{2}+T\right)^{2}}
$$

Thus, the posterior distribution can be approximated as

$$
p(\lambda \mid x) \sim N\left(\frac{(n-1 / 2)}{\left(\frac{a}{2}+T\right)}, \frac{(n-1 / 2)}{\left(\frac{a}{2}+T\right)^{2}}\right)
$$

\section{T-K Approximation}

In Lindley's approximation one requires the evaluation of third order partial derivatives of likelihood function which may be cumbersome to compute when the parameter is a vector valued parameter thus, Tierney and Kadane (1986) gave Laplace method to evaluate $E(h(\lambda) \mid x)$ as

$$
E(h(\lambda) \mid x) \cong \frac{\hat{\phi}^{*} \exp \left\{-n h^{*}\left(\hat{\lambda}^{*}\right)\right\}}{\hat{\phi} \exp \{-n h(\hat{\lambda})\}}
$$

where $-n h(\hat{\lambda})=\log P(\lambda \mid x) ;-n h^{*}\left(\hat{\lambda}^{*}\right)=\log P(\lambda \mid x)+\log h(\lambda)$

$$
\hat{\phi}^{2}=-\left[-n h^{\prime \prime}(\hat{\lambda})\right]^{-1} ; \hat{\phi}^{* 2}=-\left[-n h^{\prime *}\left(\hat{\lambda}^{*}\right)\right]^{-1}
$$

Thus, for Inverse Exponential distribution Laplace approximation for parameter $\lambda$ can be calculated as Under Quasi prior $g(\lambda)=\frac{1}{\lambda^{d}}, d \geq 0$, the posterior distribution for $\lambda$ is given in (5)

$$
-n h(\lambda)=(n-d) \log \lambda-\lambda T ;-n h^{\prime}(\lambda)=\frac{(n-d)}{\lambda}-T ; \Rightarrow \hat{\lambda}=\frac{(n-d)}{T} .
$$

Also, $-n h^{\prime \prime}(\hat{\lambda})=-\frac{T^{2}}{(n-d)}$.

Therefore $\hat{\phi}^{2}=-\left[-n h^{\prime \prime}(\hat{\lambda})\right]^{-1}=\frac{(n-d)}{T^{2}}$ or $\hat{\phi}=\frac{\sqrt{(n-d)}}{T}$,

now $-n h^{*}\left(\lambda^{*}\right)=-n h(\lambda)+\ln h(\lambda)=(n-d+1) \ln \left(\lambda^{*}\right)-\lambda^{*} T$ 


$$
\begin{aligned}
-n h^{\prime *}\left(\lambda^{*}\right)= & \frac{(n-d+1)}{\lambda^{*}}-T \Rightarrow \hat{\lambda}^{*}=\frac{(n-d+1)}{T} \\
-n h^{\prime \prime}\left(\hat{\lambda}^{*}\right)=- & \frac{T^{2}}{(n-d+1)} \\
& \hat{\phi}^{* 2}=-\left[-n h^{\prime *}\left(\hat{\lambda}^{*}\right)\right]^{-1}=\frac{(n-d+1)}{T^{2}} \text { or } \hat{\phi}^{*}=\frac{\sqrt{(n-d+1)}}{T} .
\end{aligned}
$$

Thus using (17) we have

$$
\begin{aligned}
& E(\lambda \mid x)=\frac{(n-d+1)^{1 / 2}}{(n-d)^{1 / 2}} \frac{\exp \left\{(n-d+1) \ln \hat{\lambda}^{*}-\hat{\lambda}^{*} T\right\}}{\exp \{(n-d) \ln \hat{\lambda}-\hat{\lambda} T\}}=\frac{(n-d+1)^{1 / 2}}{(n-d)^{1 / 2}} \frac{\hat{\lambda}^{*(n-d+1)} e^{-\hat{\lambda}^{*} T+\hat{\lambda} T}}{\hat{\lambda}^{(n-d)}} \\
& E(\lambda \mid x)=\left(\frac{n-d+1}{T}\right)\left(\frac{n-d+1}{n-d}\right)^{n-d+1 / 2} e^{-1}
\end{aligned}
$$

Note that the relative error (relative error to exact the posterior mean $\left(\frac{n-d+1}{T}\right)$ ) is $\left(\frac{n-d+1}{n-d}\right)^{n-d+1 / 2} e^{-1}$

Similarly $E\left(\lambda^{2} \mid x\right) \cong \frac{\hat{\phi}^{*} \exp \left\{-n h^{*}\left(\hat{\lambda}^{*}\right)\right\}}{\hat{\phi} \exp \{-n h(\hat{\lambda})\}}$,

$$
\begin{gathered}
\text { here }-n h^{*}\left(\lambda^{*}\right)=\ln \left(\lambda^{2}\right)-n h(\lambda)=(n-d+2) \ln \left(\lambda^{*}\right)-\lambda^{*} T \\
E\left(\lambda^{2} \mid x\right)=\left(\frac{n-d+2}{T}\right)^{2}\left(\frac{n-d+2}{n-d}\right)^{n-d+1 / 2} e^{-2} \\
\text { variance }=\left(\frac{n-d+2}{T}\right)^{2}\left(\frac{n-d+2}{n-d}\right)^{n-d+1 / 2} e^{-2}-\left[\left(\frac{n-d+1}{T}\right)\left(\frac{n-d+1}{n-d}\right)^{n-d+1 / 2} e^{-1}\right]^{2} .
\end{gathered}
$$

Under extension of Jeffrey's prior $g(\lambda)=\frac{1}{\lambda^{2 c_{1}}}, c_{1} \in R^{+}$, the posterior distribution for $\lambda$ is given in (8)

$$
-n h(\lambda)=\left(n-2 c_{1}\right) \log \lambda-\lambda T ;-n h^{\prime}(\lambda)=\frac{\left(n-2 c_{1}\right)}{\lambda}-T ; \Rightarrow \hat{\lambda}=\frac{\left(n-2 c_{1}\right)}{T} .
$$

Also, $-n h^{\prime \prime}(\hat{\lambda})=-\frac{T^{2}}{\left(n-2 c_{1}\right)}$. 
Therefore $\hat{\phi}^{2}=-\left[-n h^{\prime \prime}(\hat{\lambda})\right]^{-1}=\frac{\left(n-2 c_{1}\right)}{T^{2}}$ or $\hat{\phi}=\frac{\sqrt{\left(n-2 c_{1}\right)}}{T}$,

now $-n h^{*}\left(\lambda^{*}\right)=-n h(\lambda)+\ln h(\lambda)=\left(n-2 c_{1}+1\right) \ln \left(\lambda^{*}\right)-\lambda^{*} T$

$$
\begin{aligned}
& -n h^{\prime *}\left(\lambda^{*}\right)=\frac{\left(n-2 c_{1}+1\right)}{\lambda *}-T \Rightarrow \hat{\lambda}^{*}=\frac{\left(n-2 c_{1}+1\right)}{T} \\
& -n h^{\prime \prime}\left(\hat{\lambda}^{*}\right)=-\frac{T^{2}}{\left(n-2 c_{1}+1\right)}
\end{aligned}
$$

$$
\hat{\phi}^{* 2}=-\left[-n h^{\prime \prime *}\left(\hat{\lambda}^{*}\right)\right]^{-1}=\frac{\left(n-2 c_{1}+1\right)}{T^{2}} \text { or } \hat{\phi}^{*}=\frac{\sqrt{\left(n-2 c_{1}+1\right)}}{T}
$$

Thus using (17) we have

$$
\begin{gathered}
E(\lambda \mid x)=\frac{\left(n-2 c_{1}+1\right)^{1 / 2}}{\left(n-2 c_{1}\right)^{1 / 2}} \frac{\exp \left\{\left(n-2 c_{1}+1\right) \ln \hat{\lambda}^{*}-\hat{\lambda}^{*} T\right\}}{\exp \left\{\left(n-2 c_{1}\right) \ln \hat{\lambda}-\hat{\lambda} T\right\}}=\frac{\left(n-2 c_{1}+1\right)^{1 / 2}}{\left(n-2 c_{1}\right)^{1 / 2}} \frac{\hat{\lambda}^{*\left(n-2 c_{1}+1\right)} e^{-\hat{\lambda}^{*} T+\hat{\lambda} T}}{\hat{\lambda}^{\left(n-2 c_{1}\right)}} \\
E(\lambda \mid x)=\left(\frac{n-2 c_{1}+1}{T}\right)\left(\frac{n-2 c_{1}+1}{n-2 c_{1}}\right)^{n-2 c_{1}+1 / 2} e^{-1}
\end{gathered}
$$

Note that the relative error is $\left(\frac{n-2 c_{1}+1}{n-2 c_{1}}\right)^{n-2 c_{1}+1 / 2} e^{-1}$.

Similarly $E\left(\lambda^{2} \mid x\right) \cong \frac{\hat{\phi}^{*} \exp \left\{-n h^{*}\left(\hat{\lambda}^{*}\right)\right\}}{\hat{\phi} \exp \{-n h(\hat{\lambda})\}}$,

$$
\begin{gathered}
\text { here }-n h^{*}\left(\lambda^{*}\right)=\ln \left(\lambda^{2}\right)-n h(\lambda)=\left(n-2 c_{1}+2\right) \ln \left(\lambda^{*}\right)-\lambda^{*} T \\
E\left(\lambda^{2} \mid x\right)=\left(\frac{n-2 c_{1}+2}{T}\right)^{2}\left(\frac{n-2 c_{1}+2}{n-2 c_{1}}\right)^{n-2 c_{1}+1 / 2} e^{-2} \\
\text { variance }=\left(\frac{n-2 c_{1}+2}{T}\right)^{2}\left(\frac{n-2 c_{1}+2}{n-2 c_{1}}\right)^{n-2 c_{1}+1 / 2} e^{-2}-\left[\left(\frac{n-2 c_{1}+1}{T}\right)\left(\frac{n-2 c_{1}+1}{n-2 c_{1}}\right)^{n-2 c_{1}+1 / 2} e^{-1}\right]^{2} .
\end{gathered}
$$

Under the Paretol prior $g(\lambda)=b c^{b} \lambda^{-(b+1)}, c, b>0 ; \lambda>0$ where $\mathrm{b}$ and $\mathrm{c}$ are the known hyper parameters, the posterior distribution for $\lambda$ is given in (11) 


$$
-n h(\lambda)=(n-b-1) \log \lambda-\lambda T ;-n h^{\prime}(\lambda)=\frac{(n-b-1)}{\lambda}-T ; \Rightarrow \hat{\lambda}=\frac{(n-b-1)}{T} .
$$

Also, $-n h^{\prime \prime}(\hat{\lambda})=-\frac{T^{2}}{(n-b-1)}$.

Therefore $\hat{\phi}^{2}=-\left[-n h^{\prime \prime}(\hat{\lambda})\right]^{-1}=\frac{(n-b-1)}{T^{2}}$ or $\hat{\phi}=\frac{\sqrt{(n-b-1)}}{T}$,

$$
\begin{aligned}
& \text { now }-n h^{*}\left(\lambda^{*}\right)=-n h(\lambda)+\ln h(\lambda)=(n-b) \ln \left(\lambda^{*}\right)-\lambda^{*} T \\
&-n h^{\prime *}\left(\lambda^{*}\right)=\frac{(n-b)}{\lambda *}-T \Rightarrow \hat{\lambda}^{*}=\frac{(n-b)}{T} \\
&-n h^{\prime *}\left(\hat{\lambda}^{*}\right)=-\frac{T^{2}}{(n-b)} \\
& \hat{\phi}^{* 2}=-\left[-n h^{\prime *}\left(\hat{\lambda}^{*}\right)\right]^{-1}=\frac{(n-b)}{T^{2}} \text { or } \hat{\phi}^{*}=\frac{\sqrt{(n-b)}}{T} .
\end{aligned}
$$

Thus using (17) we have

$$
\begin{aligned}
& E(\lambda \mid x)=\frac{(n-b)^{1 / 2}}{(n-b-1)^{1 / 2}} \frac{\exp \left\{(n-b) \ln \hat{\lambda}^{*}-\hat{\lambda}^{*} T\right\}}{\exp \{(n-b-1) \ln \hat{\lambda}-\hat{\lambda} T\}}=\frac{(n-b)^{1 / 2}}{(n-b-1)^{1 / 2}} \frac{\hat{\lambda}^{*(n-b)} e^{-\hat{\lambda}^{*} T+\hat{\lambda} T}}{\hat{\lambda}^{(n-b-1)}} \\
& E(\lambda \mid x)=\left(\frac{n-b-1}{T}\right)\left(\frac{n-b}{n-b-1}\right)^{n-b+1 / 2} e^{-1} .
\end{aligned}
$$

Note that the relative error is $\left(\frac{n-b}{n-b-1}\right)^{n-b+1 / 2} e^{-1}$.

Similarly $E\left(\lambda^{2} \mid x\right) \cong \frac{\hat{\phi}^{*} \exp \left\{-n h^{*}\left(\hat{\lambda}^{*}\right)\right\}}{\hat{\phi} \exp \{-n h(\hat{\lambda})\}}$

$$
\begin{array}{r}
\text { here }-n h^{*}\left(\lambda^{*}\right)=\ln \left(\lambda^{2}\right)-n h(\lambda)=(n-b+1) \ln \left(\lambda^{*}\right)-\lambda^{*} T \\
E\left(\lambda^{2} \mid x\right)=\frac{(n-b-1)(n-b+1)}{T^{2}}\left(\frac{n-b+1}{n-b-1}\right)^{n-b+1 / 2} e^{-2}
\end{array}
$$


variance $=\frac{(n-b-1)(n-b+1)}{T^{2}}\left(\frac{n-b+1}{n-b-1}\right)^{n-b+1 / 2} e^{-2}-\left[\left(\frac{n-b-1}{T}\right)\left(\frac{n-b}{n-b-1}\right)^{n-b+1 / 2} e^{-1}\right]^{2}$.

Under the Inverse Levy prior $g(\lambda)=\sqrt{\frac{a}{2 \pi}} \lambda^{-\frac{1}{2}} e^{-\frac{a \lambda}{2}} ; a>0 ; \lambda>0$, where $a$ is the known hyper parameter, thus the posterior distribution for $\lambda$ is given in (14)

$$
-n h(\lambda)=\left(n-\frac{1}{2}\right) \log \lambda-\lambda\left(T+\frac{a}{2}\right) ;-n h^{\prime}(\lambda)=\frac{\left(n-\frac{1}{2}\right)}{\lambda}-\left(T+\frac{a}{2}\right) ; \Rightarrow \hat{\lambda}=\frac{(n-1 / 2)}{(T+a / 2)}
$$

Also, $-n h^{\prime \prime}(\hat{\lambda})=-\frac{(T+a / 2)^{2}}{(n-1 / 2)}$.

Therefore $\hat{\phi}^{2}=-\left[-n h^{\prime \prime}(\hat{\lambda})\right]^{-1}=\frac{(n-1 / 2)}{(T+a / 2)^{2}}$ or $\hat{\phi}=\frac{\sqrt{(n-1 / 2)}}{(T+a / 2)}$,

$$
\begin{gathered}
\text { now }-n h^{*}\left(\lambda^{*}\right)=-n h(\lambda)+\ln h(\lambda)=(n+1 / 2) \ln \left(\lambda^{*}\right)-\lambda^{*}(T+a / 2) \\
-n h^{\prime *}\left(\lambda^{*}\right)=\frac{(n+1 / 2)}{\lambda^{*}}-(T+a / 2) \Rightarrow \hat{\lambda}^{*}=\frac{(n+1 / 2)}{(T+a / 2)}
\end{gathered}
$$$$
-n h^{\prime \prime^{*}}\left(\hat{\lambda}^{*}\right)=-\frac{(T+a / 2)^{2}}{(n+1 / 2)}
$$

$$
\hat{\phi}^{* 2}=-\left[-n h^{\prime *}\left(\hat{\lambda}^{*}\right)\right]^{-1}=\frac{(n+1 / 2)}{(T+a / 2)^{2}} \text { or } \hat{\phi}^{*}=\frac{\sqrt{(n+1 / 2)}}{(T+a / 2)}
$$

Thus using (17) we have

$$
\begin{aligned}
& E(\lambda \mid x)=\frac{(n+1 / 2)^{1 / 2}}{(n-1 / 2)^{1 / 2}} \frac{\exp \left\{(n+1 / 2) \ln \hat{\lambda}^{*}-\hat{\lambda}^{*}(T+a / 2)\right\}}{\exp \{(n-1 / 2) \ln \hat{\lambda}-\hat{\lambda}(T+a / 2)\}}=\frac{(n+1 / 2)^{1 / 2}}{(n-1 / 2)^{1 / 2}} \frac{\hat{\lambda}^{*(n+1 / 2)}}{\hat{\lambda}^{(n-1 / 2)}} e^{-\hat{\lambda}^{*}(T+a / 2)+\hat{\lambda}(T+a / 2)} \\
& E(\lambda \mid x)=\left(\frac{\sqrt{(n+1 / 2)(n-1 / 2)}}{T+a / 2}\right)\left(\frac{n+1 / 2}{n-1 / 2}\right)^{n+1 / 2} e^{-1}
\end{aligned}
$$

Note that the relative error is $\left(\frac{n+1 / 2}{n-1 / 2}\right)^{n+1 / 2} e^{-1}$. 
Similarly $E\left(\lambda^{2} \mid x\right) \cong \frac{\hat{\phi}^{*} \exp \left\{-n h^{*}\left(\hat{\lambda}^{*}\right)\right\}}{\hat{\phi} \exp \{-n h(\hat{\lambda})\}}$

$$
\begin{gathered}
\text { here }-n h^{*}\left(\lambda^{*}\right)=\ln \left(\lambda^{2}\right)-n h(\lambda)=(n+3 / 2) \ln \left(\lambda^{*}\right)-\lambda^{*}(T+a / 2) \\
E\left(\lambda^{2} \mid x\right)=\frac{\sqrt{(n+3 / 2)^{3}(n-1 / 2)}}{(T+a / 2)^{2}}\left(\frac{n+3 / 2}{n-1 / 2}\right)^{n+1 / 2} e^{-2} \\
\text { variance }=\frac{\sqrt{(n+3 / 2)^{3}(n-1 / 2)}}{(T+a / 2)^{2}}\left(\frac{n+3 / 2}{n-1 / 2}\right)^{n+1 / 2} e^{-2}-\left[\left(\frac{\sqrt{(n+1 / 2)(n-1 / 2)}}{T+a / 2}\right)\left(\frac{n+1 / 2}{n-1 / 2}\right)^{n+1 / 2} e^{-1}\right]^{2} .
\end{gathered}
$$

\section{Simulation Study and Data Analysis}

\subsection{Simulation Study}

In our simulation study we have generated a sample of sizes $n=25,50$ and100 to observe the effect of small, medium, and large samples on the estimators. The results are replicated 5000 times and the average of the results has been presented in the tables. To examine the performance of Bayesian estimates for parameter of inverse exponential distribution under different approximation techniques, estimates are presented along with

\begin{tabular}{|c|c|c|c|c|c|c|c|c|c|}
\hline \multirow{2}{*}{$n$} & \multirow{2}{*}{$\lambda$} & \multicolumn{2}{|c|}{ Quasi Prior } & \multicolumn{2}{|c|}{ Extension of Jeffrey's prior } & \multicolumn{2}{|c|}{ Pareto 1 prior } & \multicolumn{2}{|c|}{ Inverse levy prior } \\
\hline & & $d=0.2$ & $d=1.2$ & $c_{1}=0.4$ & $c_{1}=1.4$ & $b=0.5$ & $b=1.5$ & $a=0.3$ & $a=1.3$ \\
\hline \multirow{3}{*}{25} & 0.5 & $\begin{array}{c}1.90423 \\
(0.19964)\end{array}$ & $\begin{array}{c}1.82744 \\
(0.19159) \\
\end{array}$ & $\begin{array}{c}1.85816 \\
(0.19480) \\
\end{array}$ & $\begin{array}{c}1.70459 \\
(0.17871) \\
\end{array}$ & $\begin{array}{c}1.80441 \\
(0.18917)\end{array}$ & $\begin{array}{l}1.727626 \\
(0.18112)\end{array}$ & $\begin{array}{c}1.85977 \\
(0.19202)\end{array}$ & $\begin{array}{c}1.79177 \\
(0.17608) \\
\end{array}$ \\
\hline & 1.5 & $\begin{array}{c}0.56752 \\
(0.02604) \\
\end{array}$ & $\begin{array}{c}0.54463 \\
(0.02499) \\
\end{array}$ & $\begin{array}{c}0.55378 \\
(0.02542) \\
\end{array}$ & $\begin{array}{c}0.50802 \\
(0.02331) \\
\end{array}$ & $\begin{array}{c}0.53777 \\
(0.02468) \\
\end{array}$ & $\begin{array}{c}0.51488 \\
(0.02363) \\
\end{array}$ & $\begin{array}{c}0.55873 \\
(0.02548) \\
\end{array}$ & $\begin{array}{c}0.55243 \\
(0.02468) \\
\end{array}$ \\
\hline & 2.5 & $\begin{array}{c}0.40159 \\
(0.00495)\end{array}$ & $\begin{array}{c}0.38541 \\
(0.00475)\end{array}$ & $\begin{array}{c}0.39188 \\
(0.00483)\end{array}$ & $\begin{array}{c}0.35949 \\
(0.00443)\end{array}$ & $\begin{array}{c}0.38055 \\
(0.00469)\end{array}$ & $\begin{array}{c}0.36435 \\
(0.00449)\end{array}$ & $\begin{array}{c}0.39578 \\
(0.00486)\end{array}$ & $\begin{array}{c}0.39261 \\
(0.00480)\end{array}$ \\
\hline \multirow{3}{*}{50} & 0.5 & $\begin{array}{c}1.95662 \\
(0.07606)\end{array}$ & $\begin{array}{c}1.91732 \\
(0.07453)\end{array}$ & $\begin{array}{c}1.93304 \\
(0.07514) \\
\end{array}$ & $\begin{array}{c}1.85446 \\
(0.07208) \\
\end{array}$ & $\begin{array}{c}1.90554 \\
(0.07407)\end{array}$ & $\begin{array}{l}1.866251 \\
(0.07255) \\
\end{array}$ & $\begin{array}{c}1.93343 \\
(0.07472) \\
\end{array}$ & $\begin{array}{c}1.89639 \\
(0.07190) \\
\end{array}$ \\
\hline & 1.5 & $\begin{array}{c}0.79184 \\
(0.00684)\end{array}$ & $\begin{array}{c}0.77594 \\
(0.00670)\end{array}$ & $\begin{array}{c}0.78230 \\
(0.00676)\end{array}$ & $\begin{array}{c}0.75050 \\
(0.00648)\end{array}$ & $\begin{array}{c}0.77117 \\
(0.00666)\end{array}$ & $\begin{array}{c}0.75527 \\
(0.00652)\end{array}$ & $\begin{array}{c}0.78520 \\
(0.00677)\end{array}$ & $\begin{array}{c}0.77902 \\
(0.00669)\end{array}$ \\
\hline & 2.5 & $\begin{array}{c}0.41094 \\
(0.00273) \\
\end{array}$ & $\begin{array}{c}0.40269 \\
(0.00267) \\
\end{array}$ & $\begin{array}{c}0.40599 \\
(0.00269) \\
\end{array}$ & $\begin{array}{c}0.38949 \\
(0.00258) \\
\end{array}$ & $\begin{array}{c}0.40022 \\
(0.00265) \\
\end{array}$ & $\begin{array}{c}0.39196 \\
(0.00260) \\
\end{array}$ & $\begin{array}{c}0.40796 \\
(0.00270)\end{array}$ & $\begin{array}{c}0.40628 \\
(0.00268)\end{array}$ \\
\hline \multirow{3}{*}{100} & 0.5 & $\begin{array}{c}2.47011 \\
(0.05355)\end{array}$ & $\begin{array}{c}2.44536 \\
(0.05302)\end{array}$ & $\begin{array}{c}2.45526 \\
(0.05323)\end{array}$ & $\begin{array}{c}2.40576 \\
(0.05216)\end{array}$ & $\begin{array}{c}2.43793 \\
(0.05286)\end{array}$ & $\begin{array}{c}2.41318 \\
(0.05232)\end{array}$ & $\begin{array}{c}2.45357 \\
(0.05302)\end{array}$ & $\begin{array}{c}2.42369 \\
(0.05182)\end{array}$ \\
\hline & 1.5 & $\begin{array}{c}0.60776 \\
(0.00556)\end{array}$ & $\begin{array}{c}0.60167 \\
(0.00551) \\
\end{array}$ & $\begin{array}{c}0.60412 \\
(0.00553)\end{array}$ & $\begin{array}{c}0.59197 \\
(0.00542)\end{array}$ & $\begin{array}{c}0.59984 \\
(0.00549) \\
\end{array}$ & $\begin{array}{c}0.59375 \\
(0.00544) \\
\end{array}$ & $\begin{array}{c}0.60538 \\
(0.00554)\end{array}$ & $\begin{array}{c}0.60354 \\
(0.00549) \\
\end{array}$ \\
\hline & 2.5 & $\begin{array}{c}0.35861 \\
(0.00141)\end{array}$ & $\begin{array}{c}0.35502 \\
(0.00139)\end{array}$ & $\begin{array}{c}0.35646 \\
(0.00139)\end{array}$ & $\begin{array}{c}0.34927 \\
(0.00136)\end{array}$ & $\begin{array}{c}0.35394 \\
(0.00138)\end{array}$ & $\begin{array}{c}0.35035 \\
(0.00137)\end{array}$ & $\begin{array}{c}0.35734 \\
(0.00140)\end{array}$ & $\begin{array}{c}0.35670 \\
(0.00139)\end{array}$ \\
\hline
\end{tabular}
posterior variances given in parenthesis in the below tables.

Table 1. Posterior Mean and Posterior Variance (In Parenthesis) under Normal Approximation 
Table 2. Posterior Mean and Posterior Variance (In Parenthesis) under T-K Approximation

\begin{tabular}{|c|c|c|c|c|c|c|c|c|c|}
\hline \multirow{2}{*}{$n$} & \multirow{2}{*}{$\lambda$} & \multicolumn{2}{|c|}{ Quasi Prior } & \multicolumn{2}{|c|}{ Extension of Jeffrey's prior } & \multicolumn{2}{|c|}{ Pareto 1 prior } & \multicolumn{2}{|c|}{ Inverse levy prior } \\
\hline & & $d=0.2$ & $d=1.2$ & $c_{1}=0.4$ & $c_{1}=1.4$ & $b=0.5$ & $b=1.5$ & $a=0.3$ & $a=1.3$ \\
\hline \multirow{3}{*}{25} & 0.5 & $\begin{array}{c}1.43544 \\
(0.24807)\end{array}$ & $\begin{array}{c}1.37982 \\
(0.23845)\end{array}$ & $\begin{array}{c}1.40207 \\
(0.24230)\end{array}$ & $\begin{array}{c}1.29083 \\
(0.22307)\end{array}$ & $\begin{array}{c}1.36313 \\
(0.22307)\end{array}$ & $\begin{array}{c}1.30752 \\
(0.22595)\end{array}$ & $\begin{array}{c}1.40701 \\
(0.23813)\end{array}$ & $\begin{array}{c}1.36924 \\
(0.21668)\end{array}$ \\
\hline & 1.5 & $\begin{array}{c}0.81404 \\
(0.02375) \\
\end{array}$ & $\begin{array}{c}0.78249 \\
(0.02283) \\
\end{array}$ & $\begin{array}{c}0.79511 \\
(0.02319) \\
\end{array}$ & $\begin{array}{c}0.73202 \\
(0.02135) \\
\end{array}$ & $\begin{array}{c}0.77303 \\
(0.02255) \\
\end{array}$ & $\begin{array}{c}0.74148 \\
(0.02163) \\
\end{array}$ & $\begin{array}{c}0.80078 \\
(0.02326)\end{array}$ & $\begin{array}{c}0.78841 \\
(0.02257)\end{array}$ \\
\hline & 2.5 & $\begin{array}{c}0.48704 \\
(0.00997)\end{array}$ & $\begin{array}{c}0.46817 \\
(0.00958)\end{array}$ & $\begin{array}{c}0.47572 \\
(0.00974)\end{array}$ & $\begin{array}{c}0.43797 \\
(0.00896)\end{array}$ & $\begin{array}{c}0.46251 \\
(0.00947)\end{array}$ & $\begin{array}{c}0.44364 \\
(0.00908)\end{array}$ & $\begin{array}{c}0.48002 \\
(0.00980)\end{array}$ & $\begin{array}{c}0.47555 \\
(0.00961)\end{array}$ \\
\hline \multirow{3}{*}{50} & 0.5 & $\begin{array}{c}2.27920 \\
(0.09236)\end{array}$ & $\begin{array}{c}2.23434 \\
(0.09054)\end{array}$ & $\begin{array}{c}2.25228 \\
(0.09127)\end{array}$ & $\begin{array}{c}2.16256 \\
(0.08764)\end{array}$ & $\begin{array}{c}2.22088 \\
(0.08999)\end{array}$ & $\begin{array}{c}2.17602 \\
(0.08818)\end{array}$ & $\begin{array}{c}2.25059 \\
(0.09065)\end{array}$ & $\begin{array}{c}2.20154 \\
(0.08693)\end{array}$ \\
\hline & 1.5 & $\begin{array}{c}0.58738 \\
(0.00807)\end{array}$ & $\begin{array}{c}0.57583 \\
(0.00791) \\
\end{array}$ & $\begin{array}{c}0.58045 \\
(0.00797) \\
\end{array}$ & $\begin{array}{c}0.55733 \\
(0.00766) \\
\end{array}$ & $\begin{array}{c}0.57236 \\
(0.00786) \\
\end{array}$ & $\begin{array}{c}0.56079 \\
(0.00771) \\
\end{array}$ & $\begin{array}{c}0.58291 \\
(0.00799)\end{array}$ & $\begin{array}{c}0.57956 \\
(0.00789) \\
\end{array}$ \\
\hline & 2.5 & $\begin{array}{c}0.33876 \\
(0.00324)\end{array}$ & $\begin{array}{c}0.33209 \\
(0.00317) \\
\end{array}$ & $\begin{array}{c}0.33476 \\
(0.00320)\end{array}$ & $\begin{array}{c}0.32143 \\
(0.00307)\end{array}$ & $\begin{array}{c}0.33009 \\
(0.00315)\end{array}$ & $\begin{array}{c}0.32343 \\
(0.00309)\end{array}$ & $\begin{array}{c}0.33643 \\
(0.00321)\end{array}$ & $\begin{array}{c}0.33531 \\
(0.00318)\end{array}$ \\
\hline \multirow{3}{*}{100} & 0.5 & $\begin{array}{c}2.14694 \\
(0.05977)\end{array}$ & $\begin{array}{c}2.12564 \\
(0.05917)\end{array}$ & $\begin{array}{c}2.13416 \\
(0.05941)\end{array}$ & $\begin{array}{c}2.09156 \\
(0.05823)\end{array}$ & $\begin{array}{c}2.11925 \\
(0.05900)\end{array}$ & $\begin{array}{c}2.09795 \\
(0.05841)\end{array}$ & $\begin{array}{c}2.13373 \\
(0.05916)\end{array}$ & $\begin{array}{c}2.11132 \\
(0.05775)\end{array}$ \\
\hline & 1.5 & $\begin{array}{c}0.75066 \\
(0.00538)\end{array}$ & $\begin{array}{c}0.74322 \\
(0.00533)\end{array}$ & $\begin{array}{c}0.74619 \\
(0.00535)\end{array}$ & $\begin{array}{c}0.73131 \\
(0.00524)\end{array}$ & $\begin{array}{c}0.74098 \\
(0.00531)\end{array}$ & $\begin{array}{c}0.73354 \\
(0.00526)\end{array}$ & $\begin{array}{c}0.74759 \\
(0.00536)\end{array}$ & $\begin{array}{c}0.74483 \\
(0.00532)\end{array}$ \\
\hline & 2.5 & $\begin{array}{c}0.44258 \\
(0.00147)\end{array}$ & $\begin{array}{c}0.43819 \\
(0.00145)\end{array}$ & $\begin{array}{c}0.43995 \\
(0.00146)\end{array}$ & $\begin{array}{c}0.43116 \\
(0.00142)\end{array}$ & $\begin{array}{c}0.43687 \\
(0.00144)\end{array}$ & $\begin{array}{c}0.43248 \\
(0.00143)\end{array}$ & $\begin{array}{c}0.44097 \\
(0.00146)\end{array}$ & $\begin{array}{c}0.44001 \\
(0.00145)\end{array}$ \\
\hline
\end{tabular}

\subsection{A Real Data Example}

In this section, we analyze the real life data set is given by Pavur et al (1992). The results recorded as the following which are the number of revolutions (in the millions) to failure of 23 ball bearings in a life test study.

$1.788,2.892,3.300,4.152,4.212,4.560,4.848,5.184,5.196,5.412,5.556,6.78,6.864,6.864,6.988,8.412$, $9.312,9.864,10.512,10.584,12.792,12.804,17.340$

Table 3. Posterior Mean and Posterior Variance (In Parenthesis) under Normal Approximation based on Data Set

\begin{tabular}{|c|c|c|c|c|c|c|c|c|}
\hline \multirow{2}{*}{$\lambda$} & \multicolumn{2}{|c|}{ Quasi Prior } & \multicolumn{2}{c|}{ Extension of Jeffrey's prior } & \multicolumn{2}{c|}{ Pareto 1 prior } & \multicolumn{2}{c|}{ Inverse levy prior } \\
\cline { 2 - 9 } & $d=0.2$ & $d=1.2$ & $c_{1}=0.4$ & $c_{1}=1.4$ & $b=0.5$ & $b=1.5$ & $a=0.3$ & $a=1.3$ \\
\hline \multirow{2}{*}{0.5} & 1.83498 & 1.75450 & 1.78669 & 1.62573 & 1.73035 & 1.64987 & 1.78923 & 1.72081 \\
& $(0.35697)$ & $(0.34131)$ & $(0.34758)$ & $(0.31626)$ & $(0.33662)$ & $(0.32096)$ & $(0.33941)$ & $(0.30127)$ \\
\hline \multirow{2}{*}{1.5} & 0.50870 & 0.48638 & 0.49531 & 0.45069 & 0.47969 & 0.45738 & 0.50033 & 0.49483 \\
& $(0.02054)$ & $(0.01964)$ & $(0.01999)$ & $(0.01819)$ & $(0.01937)$ & $(0.01846)$ & $(0.02008)$ & $(0.01950)$ \\
\hline \multirow{2}{*}{2.5} & 0.56449 & 0.53973 & 0.54964 & 0.54964 & 0.53231 & 0.50755 & 0.55500 & 0.54824 \\
& $(0.00654)$ & $(0.00625)$ & $(0.00637)$ & $(0.00579)$ & $(0.00617)$ & $(0.00588)$ & $(0.00642)$ & $(0.00632)$ \\
\hline
\end{tabular}

Table 4. Posterior Mean and Posterior Variance (In Parenthesis) under T-K Approximation based on Data Set

\begin{tabular}{|c|c|c|c|c|c|c|c|c|}
\hline \multirow{2}{*}{$\lambda$} & \multicolumn{2}{|c|}{ Quasi Prior } & \multicolumn{2}{c|}{ Extension of Jeffrey's prior } & \multicolumn{2}{c|}{ Pareto 1 prior } & \multicolumn{2}{c|}{ Inverse levy prior } \\
\cline { 2 - 9 } & $d=0.2$ & $d=1.2$ & $c_{1}=0.4$ & $c_{1}=1.4$ & $b=0.5$ & $b=1.5$ & $a=0.3$ & $a=1.3$ \\
\hline \multirow{2}{*}{0.5} & 2.08222 & 1.99476 & 2.02974 & 1.85483 & 1.96852 & 1.88106 & 2.02936 & 1.94537 \\
& $(0.42191)$ & $(0.40418)$ & $(0.41127)$ & $(0.37582)$ & $(0.39886)$ & $(0.38114)$ & $(0.40044)$ & $(0.35287)$ \\
\hline \multirow{2}{*}{1.5} & 0.57897 & 0.55465 & 0.56438 & 0.51574 & 0.54735 & 0.52304 & 0.56959 & 0.56278 \\
& $(0.01837)$ & $(0.01760)$ & $(0.01791)$ & $(0.01636)$ & $(0.01737)$ & $(0.01659)$ & $(0.01799)$ & $(0.01750)$ \\
\hline \multirow{2}{*}{2.5} & 0.32019 & 0.30674 & 0.31212 & 0.28522 & 0.30271 & 0.28926 & 0.31552 & 0.31342 \\
& $(0.01108)$ & $(0.01061)$ & $(0.01080)$ & $(0.00987)$ & $(0.01047)$ & $(0.01001)$ & $(0.01087)$ & $(0.01064)$ \\
\hline
\end{tabular}




\section{Discussion}

In this paper the focus was to study the importance of Bayesian approximation techniques. We presented approximate to Bayesian integrals of Inverse Exponential distribution depending upon numerical integration and simulation study and showed how to study posterior distribution by means of simulation study. We observe that under informative as well as non- informative priors, the normal approximation behaves well than T-K approximation, although the posterior variances in case of $\mathrm{T}-\mathrm{K}$ approximation are very close to that of normal approximation.

\section{Conclusion}

From the findings of above tables it can be observed that the large sample distribution could be improved when prior is taken into account. In both cases normal approximation as well as T-K approximation, posterior variance under Extension of Jeffrey's prior are less as compared to other assumed priors especially the Extension of Jeffrey's prior $\mathrm{c}_{1}=1.4$. We also, observe that under informative as well as non- informative priors, the normal approximation behaves well than T-K approximation, although the posterior variances in case of T$\mathrm{K}$ approximation are very close to that of normal approximation. Further we conclude that the posterior variance based on different priors tends to decrease with the increase in sample size. It implies that the estimators obtained are consistent.

It is observed that the real life data also confirms to the simulated data results. Therefore we conclude that the extension of Jeffrey's prior performs well in the IE distribution.

\section{Acknowledgement}

I am thankful to the reviewers, who made some valuable suggestions that helped me to improve my research paper.

\section{References}

[1] Ahmad SP, Ahmad, A, Khan AA. Bayesian Analysis of Gamma Distribution Using SPLUS and Rsoftwares, Asian J. Math. Stat., 2011; 4: 224-233.

[2] Ahmed AA, Khan AA, Ahmed SP. Bayesian Analysis of Exponential Distribution in S-PLUS and R Softwares, Sri Lankan Journal of Applied Statistics, 2007; 8: 95-109.

[3] Gyan Prakash. Inverted Exponential Distribution under a Bayesian Viewpoint, Journal of Modern Applied Statistical Methods, 2012; Vol. 11, No. 1, 190-202.

[4] Killer, A. Z. and Kamath, A. R. Reliability analysis of CNC machine tools, Reliability Engineering, 1982; 3:449-473.

[5] Lin, C., Duran, B., \& Lewis, T. Inverted Gamma as a Life Distribution, Microelectronics and Reliability, 1989; 29(4): 619-626.

[6] Pavur, R. J., Edgeman, R. L. and Scott, R. C. Quadratic statistics for the goodness of fit to test of inverse Gaussian distribution”, IEEE Trans. Reli., 1992;41:118-123.

[7] Sanjay Kumar Singh et. al. On the estimation of stress strength reliability parameter of inverted exponential distribution, International Journal of Scientific World, 2015; 3 (1): 98-112.

[8] Sanku Dey. Inverted Exponential Distribution as a Life Distribution Model from a Bayesian Viewpoint, Data science journal, 2007; 6: 29. 
[9] Sultan. H, Ahmad S.P. Bayesian approximation techniques of Topp-leone distribution, International Journal of Statistics and Mathematic, 2015; 2(1): 066-070.

[10] Sultan. H, Ahmad S.P. Bayesian approximation techniques for Kumaraswamy distribution, Mathematical Theory and Modeling, 2015; (5): 2225-0522.

[11] Tierney L, Kadane J. Accurate approximations for posterior moments and marginal densities, Journal of the American Statistical Association, 1986; 81: 82-86.

\section{Authors' Profiles}

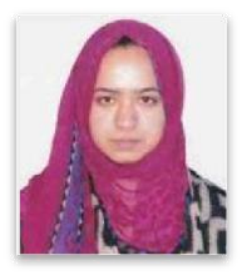

Kawsar Fatima is Research scholar at the department of Statistics, University of Kashmir, Jammu and Kashmir, India. Her research interests are in the areas of Statistical Inference including both Classical as well as Bayesian approach. She is interested in assessing the potentiality and flexibility of newly introduced probability distributions in Statistical modeling, Reliability, Order Statistics, Information theory and other different allied fields. She is also interested in studying new techniques of model construction and their implementation in introducing new versions of already existing probability distributions.

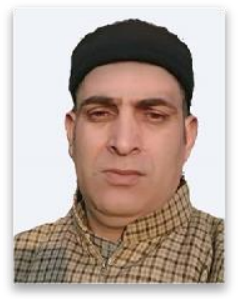

Sheikh Parvaiz Ahmad is Sr. Assistant Professor at the department of Statistics, University of Kashmir, Jammu and Kashmir, India. His research interests are in the areas of Probability distributions, Bayesian Statistics and including the classical and generalized probability distributions and Bio-statistics. He has published different research articles in different international and national reputed, indexed journals in the field of Mathematical Sciences. $\mathrm{He}$ is also refereeing of various mathematical and statistical journals especially Applied Mathematics and information Sciences, Journal of Applied Statistics and Probability, Journal of Applied Statistics and Probability Letters, International Journal of Modern Mathematical Sciences, Journal of Modern and Applied Statistical Methods and Pakistan Journal of Statistics and so on. He has presented several research articles at different international and national conferences and also attended several international and national workshops.

How to cite this paper: Kawsar Fatima, S.P Ahmad,"Bayesian Approximation Techniques of Inverse Exponential Distribution with Applications in Engineering", International Journal of Mathematical Sciences and Computing(IJMSC), Vol.4, No.2, pp.49-62, 2018.DOI: 10.5815/ijmsc.2018.02.05 